\title{
Erratum to: Effects of Short-Term Over-supplementation of Copper in Milk on Hematology, Serum Proteins, Weight Gain, and Health in Dairy Calves
}

Zahra Naseri • Mehrdad Mohri •

Mohammad R. Aslani • Gholamreza Mohammadi •

Ali A. Alavi Tabatabaee

Published online: 13 January 2012

(C) Springer Science+Business Media, LLC 2012

Erratum to: Biol Trace Elem Res (2011) 139:24-31

DOI 10.1007/s12011-010-8640-2

The original version of this article unfortunately contained a mistake. The name of Gholamreza Mohammadi is added. The correct full name of authors is shown above.

The online version of the original article can be found at http://dx.doi. org/10.1007/s12011-010-8640-2.

\section{Z. Naseri}

Center of Excellence in Ruminant Abortion and Neonatal

Mortality, School of Veterinary Medicine,

Ferdowsi University of Mashhad,

Mashhad, Iran

M. Mohri $(\bowtie) \cdot$ M. R. Aslani

Department of Clinical Sciences, and Center of Excellence

in Ruminant Abortion and Neonatal Mortality,

School of Veterinary Medicine, Ferdowsi University of Mashhad,

P.O. Box 91775-1793, Mashhad, Iran

e-mail: mohri@um.ac.ir

G. Mohammadi

Department of Clinical Sciences, School of Veterinary Medicine,

Ferdowsi University of Mashhad,

P.O. Box 91775-1793, Mashhad, Iran

\section{A. A. Alavi Tabatabaee}

Department of Veterinary Medicine,

Islamic Azad University of Bojnord,

Bojnord, Iran 